\title{
De la vue à l'ouïe. Les ekphraseis du temple de la Dive Bouteille et la tradition des progymnasmata
}

\section{Diane Desrosiers et Olivier Séguin-Brault}

\section{OpenEdition}

1 Journals

Édition électronique

URL : https://journals.openedition.org/rhetorique/1212

DOI : $10.4000 /$ rhetorique. 1212

ISSN : 2270-6909

Éditeur

UGA Éditions/Université Grenoble Alpes

Édition imprimée

ISBN : 978-2-37747-325-0

\section{Référence électronique}

Diane Desrosiers et Olivier Séguin-Brault, « De la vue à l'ouïe. Les ekphraseis du temple de la Dive Bouteille et la tradition des progymnasmata », Exercices de rhétorique [En ligne], 17 | 2021, mis en ligne le 25 novembre 2021, consulté le 29 novembre 2021. URL : http://journals.openedition.org/rhetorique/ 1212 ; DOI : https://doi.org/10.4000/rhetorique.1212

Ce document a été généré automatiquement le 29 novembre 2021.

\section{(c) (i) (9)}

Les contenus de la revue Exercices de rhétorique sont mis à disposition selon les termes de la Licence Creative Commons Attribution - Pas d'Utilisation Commerciale - Partage dans les Mêmes Conditions 4.0 International. 


\title{
De la vue à l'ouïe. Les ekphraseis du temple de la Dive Bouteille et la tradition des progymnasmata
}

\author{
Diane Desrosiers et Olivier Séguin-Brault
}

\section{Introduction. L'ekphrasis rabelaisienne, entre héritage et transgression}

1 On connaît le rôle majeur joué par les manuels d'exercices préparatoires de rhétorique dans la formation de l'élite lettrée en France durant la première modernité. Entre les $\mathrm{XVI}^{\mathrm{e}}$ et $\mathrm{XVII}^{\mathrm{e}}$ siècles, les progymnasmata (ou prceexercitamenta) firent l'objet de plus d'une centaine d'éditions, de commentaires savants, de traductions et de réimpressions qui connurent une large diffusion en Europe ${ }^{1}$. Pierre angulaire de l'enseignement rhétorique dispensé dans les collèges jésuites, le cursus progymnasmatique menait l'étudiant à travers une séquence de 14 exercices préliminaires, ordonnés de façon croissante en fonction de leur complexité (de la fable à la défense de loi), en vue de parvenir à la composition d'une declamatio. La pratique des exercices préliminaires dans la littérature de l'Ancien Régime témoigne de la fortune inédite de ces manuels pédagogiques et du rayonnement ininterrompu dont jouissait l'ars bene dicendi dans les cercles intellectuels, au moins jusqu'à la fin du xviII siècle. La réception des progymnasmata dans l'œuvre de Rabelais illustre en particulier l'actualisation des modèles rhétoriques hérités de l'Antiquité gréco-latine ${ }^{2}$.

2 À travers une série de déplacements des règles de composition prescrites par les théoriciens antiques et renaissants, les textes rabelaisiens participent de surcroit au recyclage parodique des différents genres rhétoriques. Les séquences descriptives qui jalonnent la geste pantagruéline opèrent par exemple un décalage vis-à-vis de la théorie classique de l'ekphrasis ${ }^{3}$, qui correspond à la définition que propose Paul Zumthor de la parodie: "une opération seconde qui prend son point de départ dans une technique donnée, mais la dénature ${ }^{4} »$. 
3 Dans les descriptions de l'abbaye de Thélème ${ }^{5}$ (Gargantua, ch. LIII-LVIII ${ }^{6}$ ) et de l'anatomie de Quaresmeprenant ${ }^{7}$ (Quart livre, ch. XxX-XxxII), l'ordre du parcours visuel contrevient en effet à la mécanique traditionnellement préconisée par les théoriciens ${ }^{8}$. Comme le note Philippe Hamon, en adoptant dans ces deux passages un mouvement allant de l'intérieur vers l'extérieur et du bas vers le haut, Rabelais « parodie l'ordre normal du genre descriptif (blason, etc.) ${ }^{9} »$. Le traitement de la théorie de l'ekphrasis dans les cinq Livres s'inscrit ainsi dans la lignée des préoccupations soulevées par Patricia EichelLojkine au seuil de son ouvrage sur la parodie à la Renaissance :

S'il est vrai que la finalité de Rabelais est bien plus de revitaliser l'écriture en «recyclant» des discours tout faits et des genres fixés par la tradition que d'illustrer tel ou tel système d'idées, alors il faut commencer par étudier de près les détournements qu'il fait subir aux modèles discursifs et narratifs dont il hérite, et déterminer l'orientation de ces détournements ${ }^{10}$.

4 À l'instar de ces «ekphraseis inversées ", la traversée du temple de la Dive Bouteille, dernier passage du livre apocryphe attribué à Rabelais et composé de brouillons agencés par les compilateurs de son œuvre, comme l'a montré Mireille Huchon ${ }^{11}$, forme un exercice descriptif exemplaire conduit à rebours des prescriptions énoncées par les auteurs de progymnasmata. La séquence des chapitres XXXVI-XLVI du Cinquiesme livre constitue non seulement un micro-récit de l'initiation bachique, sur le modèle de l'initiation aux « mystères d'amour » du Songe de Poliphile, mais encore une initiation à la parole dont le mouvement général inverse la logique descriptive en tentant de «transformer les spectateurs en auditeurs ${ }^{12}$ ». Suivons donc Pantagruel, Panurge et frère Jean au cœur du temple bachique : «Boutons, boutons, passons outre»!

\section{Ekphrasis et parodie : le cas de la traversée du temple de la Dive Bouteille}

5 Au terme de la quête pour obtenir le mot de la Dive Bouteille amorcée à la fin du Tiers livre, les voyageurs de la Thalamège parviennent à leur destination finale et entreprennent la descente vers le temple de la Pontife Bacbuc, l'interprète de la «bouteille trimegiste ». Au seuil de l'entrée du sanctuaire, la lanterne qui sert de guide aux voyageurs interrompt le dialogue de Panurge et frère Jean pour signaler l'amorce du développement « énargique ${ }^{13}$ » : « Icy fut le propos interrompu par nostre splendide lanterne nous remonstrant, que là estoit le lieu, auquel convenoit favorer et par suppression de parolles, et taciturnité de langues » $(C L, 813)$. L'admonition de la lampe qui enjoint les personnages à se taire participe au glissement de la narratio vers la descriptio. L'exhortation finale de Panurge traduit d'ailleurs le passage vers un autre lieu du discours : « Passons donques, dist Panurge, et donnons de la teste à travers tous les diables. " (CL, 813). La prise en charge définitive du parcours ambulatoire est marquée par l'adresse de la lanterne qui commande aux trois personnages d'être attentifs et de "n'avoir frayeur ne peur aucune» (CL, 814). L'attention du lecteurauditeur captée, la visite peut enfin débuter.

6 La séquence ekphrastique qui suit, conduite selon les préceptes de l'art oratoire, accumule les descriptions visuelles. Du point de vue de la mécanique descriptive, cette séquence constitue un exercice exemplaire de composition ekphrastique. Le relais de la pause descriptive est d'emblée indiqué par un changement de temps de verbe, du passé simple (« temps signalétique de l'effet-récit ${ }^{14} »$ ) à l'indicatif imparfait, et l'accumulation 
de verbes de vision: «je projettay ma veuë entre les portes » $(C L, 815)$, «j'apperçeu » $(C L, 815)$, «j'apperçeu d'avantage » $(C L, 815)$, « je veis » $(C L, 815)$ et « jettay mes yeux à la contemplation du manifique temple » $(C L, 816)$. Après avoir observé la composition du vignoble et de l'arc menant au temple souterrain (ch. XxxIII), le regard descripteur se dirige vers le portail et le mécanisme magnétique des portes (ch. XxxvI), se pose ensuite sur le pavé du temple (ch. XxxvII) et la «mirifique emblemature» de sa voûte (ch. XXXVIII-XXXIX), avant d'atteindre la lampe finement gravée (ch. XL-XLI) et la fontaine monumentale trônant au «lieu moyen du temple» (ch. XLI-XLII). Le parcours ambulatoire, qui s'amorce sur le mouvement d'ouverture des portes, se clôt sur la sortie du jet de la fontaine et nous mène du jardin de Bacchus au cœur du sanctuaire, correspond à la dynamique périégétique ${ }^{15}$ préconisée par les rhéteurs pour la composition de l'ekphrasis. La visite du temple, ponctuée par des déictiques spatiaux qui orientent le parcours visuel, nous conduit ainsi de l'extérieur vers l'intérieur. La description de la fontaine, dernier élément de la séquence, traduit elle-même la cinétique circumambulatoire vers le cœur de l'objet " placé sous les yeux ». Le discours " évident » expose d'abord " [1]a premiere colomne, sçavoir est, celle laquelle à l'entrée du temple s'objettoit à nostre veuë » $(C L, 825)$, avant de passer en revue chacune des sept colonnes disposées en cercle. Le regard descriptif circule une seconde fois autour de la fontaine en observant les sculptures qui trônent " au dessus du chapiteau » (CL, 825) des colonnes. Il se porte ensuite sur « les bases des colomnes, les chapiteaux, les architraves, zoophores et cornices" $(C L, 826)$, sur les " arceaux entre les colomnes » $(C L, 826)$, sur le dessus des « arces et chapiteaux de colomne » $(C L, 826)$ et accède enfin «[d]edans la corpulance d'icelle» $(C L, 826)$ et «[s]us le sommet de la croppe susdite, correspondant au centre de la fontaine » $(C L, 827)$. Le son produit par la fontaine, " comme de loin venant et soubterrain" ( $C L, 827)$, emprunte le même mouvement ascendant et inspire à Bacbuc une analogie physiologique : «telle qu'est en la veine cave, au lieu qu'elle entre le dextre ventricule du cœur, est ceste sacrée fontaine excolée, et par icelle armonie telle, que elle monte jusques à la mer de vostre monde » $(C L, 828)$.

7 Le mouvement circulaire adopté pour la description du temple s'exprime également à travers une série d'objets sphériques, à l'instar de la " grande lampe ronde » $(C L, 822)$ retenue à un anneau d'or et composée de quatre anneaux contenant une boule vide, ou encore de la fontaine qui « [p]ar dedans estoit ronde exactement» (CL, 824). Le jet d'eau (ou plutôt le jet d'«o») de la fontaine et les deux lignes en spirale que forment ses canaux renvoient eux-mêmes à la cinétique du parcours ambulatoire. La description de la fontaine s'effectue en effet grâce à deux déplacements circulaires ascendants qui prennent pour objet les sept colonnes et leurs chapiteaux. La circumambulation, mouvement symbolique dans plusieurs cultes religieux, est même mise en scène lors du rituel pour obtenir le mot de la Dive Bouteille lorsque Panurge chemine neuf fois autour de la fontaine et danse trois fois autour de celle-ci. Le motif de la circularité apparaît également dans l'image de la «sphere intellectuale [...] que nous appellons dieu » $(C L, 839)$, de même que dans la structure de l'ekphrasis, à travers la configuration du temple de Bacchus. La chapelle dans laquelle les visiteurs terminent leur parcours est en effet de forme circulaire, tout comme le sanctuaire de Fortuna Primigenia à Palestrina mentionné au chapitre XxxvII, avec sa tholus sommitale, modèle exemplaire de la typologie architecturale du temple rond ${ }^{16}$. Le plan architectural du sanctuaire nous permet en outre d'établir un parallèle entre Thélème bâtie en forme d'hexagone avec une tour ronde à chaque angle et le temple du Cinquiesme livre sur la base de leur 
géométrie. Comme l'écrit l'auteur du De re cedificatoria, l'architecte italien Leon Battista Alberti, la nature favorise les modèles circulaire et hexagonal :

La nature aime tout particulièrement les objets ronds, comme il ressort avec évidence de ce qui existe, naît ou se produit sous cette forme. Ai-je besoin de mentionner l'orbe du monde, les astres, les arbres, les animaux et leurs nids, etc. que la nature a tous voulus ronds? Mais nous voyons que la nature aime également les objets hexagonaux. En effet, les abeilles, les frelons et autres insectes de ce genre ne savent construire les loges de leurs théâtres qu'hexagonales ${ }^{17}$.

La rotondité est la forme privilégiée par Alberti pour la construction d'un temple voué au culte bachique: «[les Anciens] jugaient bon que le Soleil et le vénérable Bacchus aient un temple rond ${ }^{18} »$. L'architecture du temple de la Dive reproduit donc la circularité de la disposition rhétorique, tout comme le texte reproduit dans sa matérialité même la forme circulaire de la bouteille grâce au calligramme du chapitre $\mathrm{XLIV}^{19}$. L'apostrophe liminaire du chant entonné par Bacbuc («O Bouteille») fait d'ailleurs écho au goulot représenté par trois cercles concentriques dans l'édition du Cinquiesme livre retenue par Mireille Huchon comme texte de base aux Euvres complètes (fig. 1), de même qu'à l'ovale de la bouteille illustrant l'édition lyonnaise de 1564 (fig. 2). La répétition du sixain, placé au début et à la fin du poème, accentue par ailleurs la circularité du calligramme.

$\mathrm{Au}$ cœur du parcours visuel, le narrateur introduit une mise en abyme de l'acte descriptif qui signale l'amorce d'un nouveau développement ekphrastique : "avant qu'entrer à l'exposition de la Bouteille, je vous descriray la figure admirable d'une Lampe » (CL, 821).

Au milieu de la voulte estoit un anneau d'or massif attaché, de la grosseur de plein poing: auquel pendoient de grosseur peu moindre, trois chesnes bien artificiellement faites, lesquelles deux pieds et demy en l'air comprenoient en figure triangle une lame de fin or, ronde, de telle grandeur que le diametre excedoit deux coudées, et demye palme. En icelle estoient quatres boucles ou pertuys : en chascune desquelles estoit fixement retenue une boule vuyde, cavée par le dedans, ouverte du dessus, comme petite Lampe, ayant en circonferance environ deux palmes, et estoient toutes de pierres bien precieuses : l'une d'Amethyste l'autre de Carboucle Lybien, la tierce d'Opalle, la quarte d'Anthracite ${ }^{20}$. (CL, 822)

9 La descriptio se poursuit avec l'examen minutieux de cette «lampe memorable » ( $C L$, 823) et de sa fresque gravée en relief, accumulant les détails (dimensions, couleurs et matériaux) qui permettent une vive représentation de l'objet dans l'imaginaire du lecteur en le plaçant "sous les yeux» ( $\dot{v} \pi^{\prime}$ ö $\left.\psi v v\right)$. Au terme de cette description, le narrateur observe que le scintillement de la fontaine répond à l'éclat des «lampes cy dessus descriptes » $(C L, 827)$. Les deux occurrences du verbe "décrire » qui ceignent l'exposition détaillée de la lampe et de la fontaine possèdent une fonction métadiscursive et réitèrent la dimension rhétorique de l'exposé énargique. Le dernier épisode du Cinquiesme livre emprunte ainsi les lieux de l'ekphrasis et constitue le lieu privilégié pour la mise en scène de l'acte descriptif.

\section{Une ekprasis « au contraire de toutes aultres »}

10 À l'aube du développement descriptif entrepris au chapitre xxxvi, une série de décalages permettent toutefois de situer la séquence du Cinquiesme livre à rebours du modèle aphthonien. Dans les exemples d'ekphraseis fournis par les théoriciens de l'Antiquité21 , le discours énargique comporte un exorde et un épilogue qui introduisent 
le mouvement allant du général au particulier. Dans la description de l'acropole d'Alexandrie qui apparaît dans les Progymnasmata d'Aphthonios, "best-seller européen » édité à maintes reprises dans la première moitié $d u \mathrm{XVI}^{\mathrm{e}}$ siècle ${ }^{22}$, la périégèse débute par des considérations d'ordre onomastique et se termine sur une formule de prétérition propre au registre du topos de l'indicible :

Et, s'il est vrai que c'est la cour d'Athènes qui renferme son acropole, la citadelle qu'Alexandre a désignée comme celle de sa propre cité, il l'a rendue conforme au nom qu'il lui a donné : il l'a établie en effet sur le point culminant de sa ville et elle porte plus légitimement le nom d'acropole que celle dont s'enorgueillit Athènes, car elle possède la qualité exprimée par ce $\operatorname{mot}^{23}$.

Mais cette beauté est trop grande pour qu'on puisse l'exprimer. Et ce qui a pu être omis ne fait qu'ajouter au merveilleux, car il n'a été omis que parce qu'il était indicible ${ }^{24}$.

11 Le récit de la descente vers le temple de la Dive Bouteille, au seuil du parcours descriptif du Cinquiesme livre, assume la fonction d'épilogue. En route vers l'oracle de la Dive Bouteille, Pantagruel énumère ainsi les villes qui reçurent le nom de leur fondateur, à l'instar d'« Alexandre à Alexandrie, Constantin à Constantinople, Pompée à Pompéiopolis en Cilicie, Adrian à Adrianople, Canaan aux Cananeens, Saba aux Sabeians, Assur aux Assyriens" (CL, 811). Ce recensement fait écho aux propos préliminaires de l'ekphrasis d'Aphthonios où le rhéteur mentionne qu'Alexandre donna son nom à la ville d'Alexandrie. Le même principe apparaît d'ailleurs dans la description du Pantagruélion, aux chapitres L-LI du Tiers livre: "Je trouve que les plantes sont nommées en diverses manieres. Les unes ont prins le nom de celluy qui premier les inventa, congneut, monstra, cultiva, aprivoisa, et appropria, comme Mercuriale de Mercure : Panacea de Panace [...]» $(T L, 503)$. Or, dans le Cinquiesme livre Pantagruel prend la peine de préciser la raison du nom de Chinon, première ville du monde : "Cayn fut premier bastisseur de villes : vray doncques semblable est, que la premiere, il de son nom nomma Cainon» $(C L, 810)$. Cependant, a contrario des cités nommées d'après leur illustre fondateur, l'île où prend fin la navigation de l'équipée de la Thalamège affiche un anonymat complet ${ }^{25}$.

Le trajet menant les personnages au temple de la Dive opère également un décalage visà-vis de l'exemplum d'Aphthonios. Au mouvement ascendant du chemin vers le Sérapéum se substitue la dynamique inverse de la catabase vers les profondeurs du temple de la Dive, accentuée par la répétition du mouvement au début de trois chapitres consécutifs: "[d]escendant Panurge en terre» (CL, 808), "[a]insi descendismes sous terre » $(C L, 810)$ et «[d]epuis descendismes un degré marbrin sous terre » $(C L, 811)$. Si le nombre de marches semble dans un premier temps correspondre à la «mesure parfaite ${ }^{26}$ » de cent, comme pour l'acropole d'Alexandrie, le compte des marches atteint après révision le «nombre fatal» $(C L, 811)$ de cent huit. Mais ce n'est pas tout. Le sentiment de protection assuré par l'acropole d'Alexandrie, érigée sur les hauteurs de la cité et établie pour la sécurité de la ville à laquelle elle sert de rempart, comme le signale Aphthonios, fait également l'objet d'un renversement dans le texte rabelaisien. Une fois «en seureté » dans l'enceinte du complexe, Panurge est en effet terrifié et croit descendre aux Enfers: «Dame mirifique, je vous prie de cœur contrit, retournons arriere: par la mort bœuf, je meurs de malle peur» $(C L, 812)$. L'accumulation de ces éléments placés en marge de l'ekphrasis rabelaisienne confère un certain vernis parodique à la séquence descriptive. 
13 C'est toutefois dans le renversement de la logique descriptive qui vise à «transformer l'auditeur en spectateur " que prend véritablement forme le recyclage parodique des modèles rhétoriques dans le texte rabelaisien. À la toute fin du parcours ambulatoire, le «coulement et laps de la fontaine » $(C L, 827)$ qui sollicite le sens auditif prélude au terme de la pause descriptive et au retour de la parole narrative. La transition du sens visuel au sens auditif est en quelque sorte anticipée par Pantagruel lorsqu'il signale, en regardant le centre de la fontaine : " peu s'en faillit, que perdissions la veuë » $(C L, 827)$. De la même façon que la Lampe a interrompu le dialogue des personnages pour faire place à la pause descriptive au chapitre Xxxv, Bacbuc commande aux trois visiteurs de diriger leur attention sur le ruissellement de la fontaine :

Nous avions iceux [le coulement et laps de la fontaine] consideré, ailleurs tournions notre veuë, quant Bacbuc nous commanda entendre à l'exciture de l'eau, lors entendismes un son à merveille harmonieux, obtus toutefois, et rompus, comme de loin venant et soubterrain. En quoy plus nous sembloit delectable, que si apert eus esté, et de prés ouy. De sorte qu'autant comme par les fenestres de nos yeux, nos esprits s'estoient oblectez à la contemplation des choses susdites, autant en restoitil aux aureilles à l'audiance de ceste harmonie. $(C L, 827)$

La réminiscence graduelle de l'ouie annonce la réintroduction de la narratio dans le récit et laisse bientôt place à une effusion de paroles : « [a]donc nous dist Bacbuc » (CL, 827-828), «[p]uis commanda » (CL, 828), «[p]uis nous interrogua» $(C L, 828)$. La description du temple de la Dive est d'ailleurs suivie de quatre chapitres qui accumulent les formes discursives et donnent lieu à un excès d'élocution insoutenable. L'effet de la « fureur poëtique » qui anime les visiteurs du temple les conduit à rimer de façon paroxysmique. La séquence descriptive apparaît ainsi comme le point de départ d'un mouvement d'émancipation élocutoire qui concentre à l'échelle de six chapitres le microrécit d'une « initiation à la parole ». Le mouvement de crescendo perceptible dans la séquence descriptive du Cinquiesme livre se distingue donc de la dissolution de la parole narrative mise en scène dans l'excipit descriptif du Gargantua où la narratio est interrompue par la descriptio de l'abbaye de Thélème et l'énigme en prophétie, laquelle " n'est autre chose qu'une déscription ${ }^{27}$ ", comme le signale Thomas Sébillet dans son Art poetique françoys. La parole narrative ne refait surface que l'instant d'une brève exégèse de l'« inscription mise sus la grande porte de Theleme ", laquelle n'est autre qu'une « description du Jeu de Paulme » $(G, 153)$ selon frère Jean.

La description de la mosaïque du temple de la Dive Bouteille illustre plus particulièrement «l'avènement de la parole ${ }^{28} »$. Dans l'hypotypose des chapitres XXXVIII-XXXIX, les sens visuel et auditif se confondent à l'instar des œuvres d'« Apelles, Aristides Thebain, et autres qui ont painct les tonnerres, esclairs, foudres, vents, paroles » $(C L, 820)$. Cette ekphrasis qui accumule les éléments sonores s'inscrit dans la tradition des "peintures parlantes ${ }^{29}$ " inaugurée par les Eikones de Philostrate ${ }^{30}$, véritable «référence pour la composition d'une ecphrasis [dans la première modernitée $]^{31}$ ». Suite à l'examen du portail où apparaît l'adage «Ev oív $\omega \dot{\alpha} \lambda \eta \dot{\theta} \theta \varepsilon i \alpha$ » (soit, en latin, in uino ueritas), la description se dirige sur le mécanisme magnétique des portes du temple qui est «merveilleux entendre » $(C L, 814)$. Dans cette « description de manière ${ }^{32}$ ", le sens auditif sollicité par l'ouverture des portes se substitue rapidement au regard descripteur :

Soudainement les deux portes, sans que personne y touchast, de soy-mesme s'ouvroient, et s'ouvrant firent, non bruit strident, non fremissement horrible, comme font ordinairement portes de bronze, rudes et pesantes, mais doux et gratieux murmur, retentissant par la voulte du temple, duquel soudain Pantagruel 
entendit la cause, voyant sous l'extremité de l'une et l'autre porte, un petit cylindre, lequel [...] par son frottement faisoit ce doux et harmonieux murmur ${ }^{33}$. $(C L, 814)$ où est représentée la victoire de Bacchus sur les Indiens. Dans la description du cortège de Bacchus résonne d'abord le bruit des «petits boucliers legers, sonnans et bruyans quant on y touchoit [...], comme de tabourins et de tymbons» (CL, 818), des «jeunes gens [...] tousjours chantans » $(C L, 819)$ et de l'évocation du mot Evohé sur laquelle se termine le chapitre XxxviII. La représentation de l'affrontement sur laquelle se poursuit la description de l'œuvre d'art s'anime ensuite d'une cacophonie de cris, de chants et de percussions amplifiée par le «tumulte horrible des Bacchides» (CL, 820) et du tintamarre de «joyeux Epinicies, et petites chansons villatiques et dithyrambes resonnans » $(C L, 821)$ :

Les Satyres Capitaines, Sergens de bandes, Caps d'Escadre, Corporals, avec cornaboux sonnant les orties furieusement tournoyoient autour de l'armée à saux de chevres, à bons, à pets, à ruades, et penades, donnans courage aux compagnons de vertueusement combatre : tout le monde en figure cryoit Evohé. Les Menades premieres faisoient incursion sur les Indians avec cris horribles, et sons espouvantables de leurs tymbous et boucliers : tout le Ciel en retentissoit, comme designoit l'Emblemature. [...] Là eussiez veu Silenus son Asne [...] la gueule bée comme s'il brailloit, et braillant martiallement [...] sonnast l'assaut. (CL, 820).

On retrouve plusieurs de ces éléments sonores dans Le Songe de Poliphile et la prolalia de Lucien de Samosate ${ }^{34}$. Or, à la différence des hypotyposes empruntées à Colonna et à Lucien, les descriptions sonores du Cinquiesme livre s'inscrivent dans une progression du mutisme au chant poétique. La particularité de la séquence du temple de la Dive Bouteille réside en effet dans le mouvement croissant d'émancipation oratoire qui débute avec la suppression des paroles au seuil du temple, se poursuit avec la réintroduction partielle du sens auditif par le jeu des sonorités de la fresque bachique et prend fin avec le jaillissement de la parole menant à la «fureur poëtique». Les chants entonnés par Panurge, Pantagruel et frère Jean prennent d'ailleurs la forme d'un trialogue : «Trinquons, dist Panurge, de par le bon Bacchus» (CL, 834), «Pantagruel reprend frere Jean, et luy dit » (CL, 835), « Panurge continue son propos, et dit» (CL, 836), «Frere Jean respondit en fureur, et dist » $(C L, 837)$ et «Panurge $[. .$.$] respondit »$ (CL, 838). En s'efforçant de "transformer les spectateurs en auditeurs», le crescendo de la vue à l'ouie mis en scène dans l'épisode de la Dive Bouteille procède ultimement au renversement de la logique descriptive. Dans ce passage, le regard rhétorique est constamment subordonné au sens auditif. L'oreille demeure ainsi l'organe privilégié comme instrument du docere ${ }^{35}$, comme Pantagruel en fait la démonstration dans le Tiers livre :

Nature me semble non sans cause nous avoir formé aureilles ouvertes, n'y appousant porte ne clousture aulcune, comme a faict es œilz, langue, et aultres issues du corps. La cause je cuide estre, affin que tous jours, toutes nuyctz, continuellement, puissions ouyr : et par ouye perpetuellement aprendre : car c'est le sens sus tous aultres plus apte es disciplines. (TL, 401)

18 À l'issue de cette séquence, les trois visiteurs auront donc fait l'expérience d'une initiation à la parole qui correspond à la finalité du cursus progymnasmatique, laquelle vise à développer les compétences oratoires nécessaires à la pratique déclamatoire. Ce parcours coïncide d'ailleurs avec le passage de l'obscurité à la lumière, faisant écho au parcours initiatique de Panurge, frère Jean et Pantagruel pour recevoir le mot de la 
Dive Bouteille. De l'anti-abbaye de frère Jean au temple souterrain du Cinquiesme livre, la récupération parodique et le déplacement ludique des formes discursives confèrent ainsi aux modèles anciens un sens renouvelé qui invite à une lecture à plus hault sens des descriptions architecturales.

\section{ANNEXES}

\section{Figure 1}

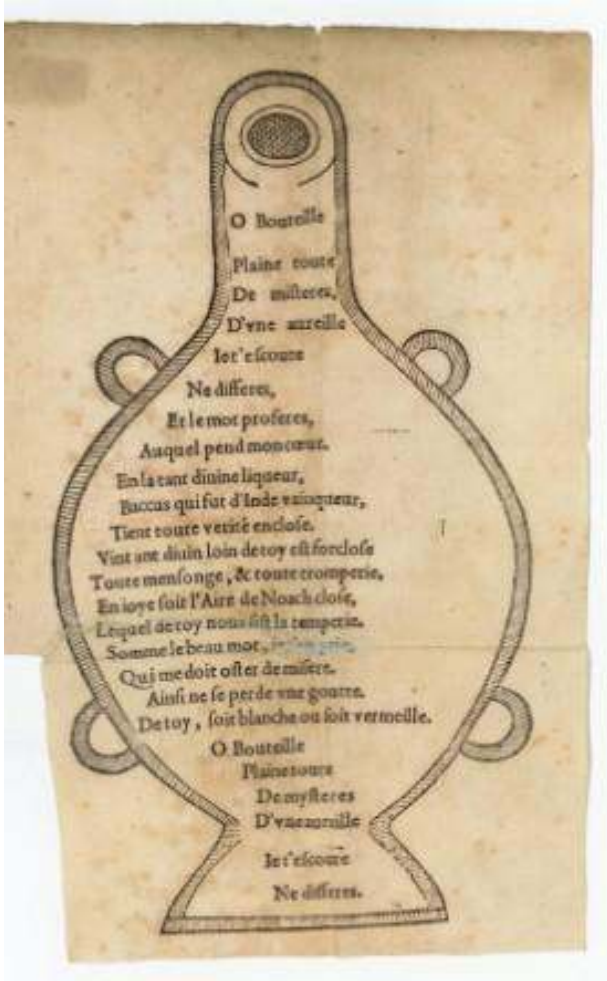

François Rabelais, Cinquiesme et dernier livre des faicts et dicts héroïques du bon Pantagruel, 1565. (CBNF)

\section{Figure 2}




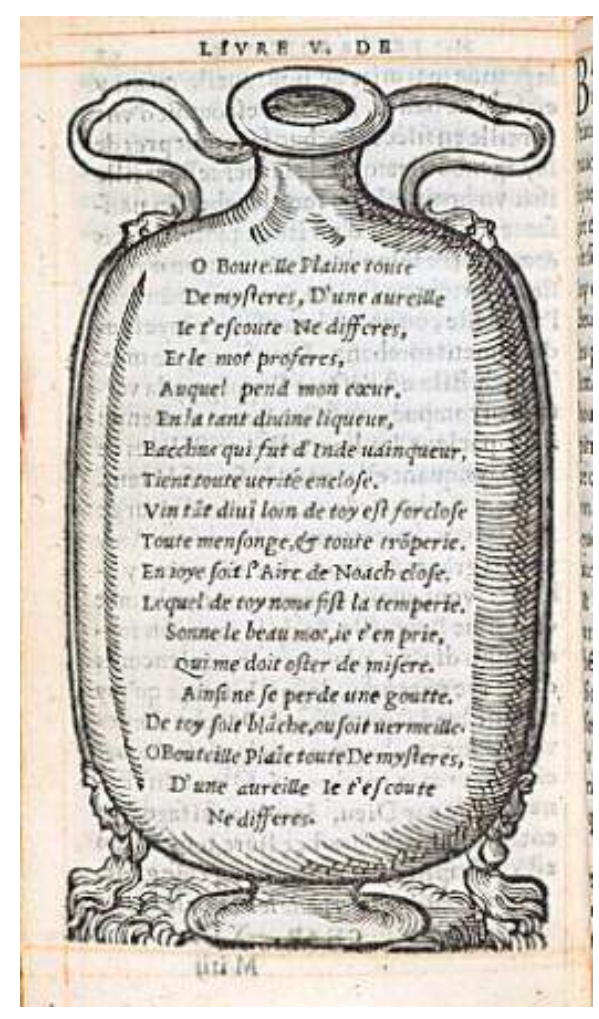

François Rabelais, Les œuvres de M. François Rabelais..., Lyon, 1564. (@Bibliothèque municipale de Tours, cliché François Joly)

\section{NOTES}

1. Pour une liste complète des manuels de Progymnasmata et de leurs commentaires édités à la Renaissance, on pourra consulter la bibliographie de P.Chiron: «Les progymnasmata de l'Antiquité gréco-latine ", Lustrum, n 59, 2017, p. 7-130.

2. Sur la tradition des exercices préparatoires dans l'œuvre de Rabelais, voir notamment D. Desrosiers, "An muri faciendi": la pratique des progymnasmata dans l'œuvre de François Rabelais ", dans P. Chiron et B. Sans (dir.), Les progymnasmata en pratique, de l'Antiquité à nos jours / Practicing the Progymnasmata, from Ancient Times to Present Days, Paris, Éditions Rue d'Ulm, 2020, p. 218-226 ; id., " "An muri faciendi”. Les progymnasmata dans le Pantagruel de Rabelais ", dans R. Menini et al. (dir.), Mélanges Mireille Huchon, Paris, Classiques Garnier, à paraître ; id., « Le Tiers Livre de Rabelais et la tradition des progymnasmata", dans M. Huchon et al. (dir.), Inextinguible Rabelais, Paris, Classiques Garnier, 2020, p. 305-315.

3. La composition d'une description (ekphrasis) figure au rang des exercices préliminaires dans le programme progymnasmatique. Considéré comme l'un des exercices les plus techniques, l'ekphrasis apparaît généralement vers la fin de la formation rhétorique. Loin d'être restreint à la description d'une œuvre d'art, le développement ekphrastique embrasse tout objet susceptible d'être exposé «de manière évidente ». Pour une étude détaillée de la théorie classique de l'ekphrasis, voir notamment R. Romagnino, Théorie(s) de l'ecphrasis entre Antiquité et première modernité, Paris, Classiques Garnier, 2019; R. Webb, «Ekphrasis in the Classroom and in the Progymnasmata ", dans P. Chiron et B. Sans (dir.), Les Progymnasmata en pratique, op. cit., p. 150-162 ; et de la même auteure, Ekphrasis, Imagination and Persuasion in Ancient Rhetorical Theory and Practice, Farnham, Ashgate, 2009 [2012] ; P. Galand-Hallyn, Le reflet des fleurs : description et métalangage poétique d'Homère à la Renaissance, Genève, Droz, 1994 ; et de la même auteure, Les yeux de l'éloquence : poétiques humanistes de l'évidence, Orléans, Paradigme, 1995. 
4. P. Zumthor, Langue et techniques poétiques à l'époque romane (XI ${ }^{e}-\mathrm{XIII}{ }^{e}$ siècle), Paris, Klincksieck, 1963, p. 93. Gérard Genette propose quant à lui la définition suivante (dans Palimpsestes. La littérature au second degré, Paris, Seuil, 1982, p. 20) : " parôdia, ce serait (donc ?) le fait de chanter à côté, donc de chanter faux, ou dans une autre voix, en contrechant - en contrepoint -, ou encore de chanter dans un autre ton : déformer, donc, ou transposer une mélodie. »

5. Les modalités de ces descriptions ont été rapprochées de la «méthode descriptive » employée par Francesco Colonna dans l'Hypnerotomachia Poliphili, de la poétique du temple des Grands Rhétoriqueurs et de la tradition rhétorique de l'ekphrasis. Sur ce sujet, voir plus particulièrement G. Polizzi, "Thélème ou l'éloge du don: le texte rabelaisien à la lumière de l'Hypnerotomachia Poliphili", Réforme, Humanisme, Renaissance, $\mathrm{n}^{\circ} 25,1987$, p. 39-59; D. Desrosiers, "L'abbaye de Thélème et le temple des Grands Rhétoriqueurs ", dans M. Simonin (dir.), Rabelais pour le XXI siècle. Actes du colloque du Centre d'Études Supérieures de la Renaissance (Chinon-Tours, 1994), Genève, Droz (Études rabelaisiennes, vol. XXXIII), 1998, p.241-248; et O. Séguin-Brault, "Thélème: parcours descriptif et mécanique rhétorique ", L'Année rabelaisienne, $\mathrm{n}^{\circ}$ 2, 2018, p. 285-295.

6. F. Rabelais, Gargantua, dans Euvres complètes, M. Huchon (éd.), Paris, Gallimard, 1994, p. 139-153. Toutes les citations ultérieures aux textes rabelaisiens seront tirées de cette édition; la référence apparaîtra dans le corps du texte entre parenthèses.

7. L'ordre de la description anatomique de Quaresmeprenant a été étudié par M.-M. Fontaine, "Quaresmeprenant: l'image littéraire et la contestation de l'analogie médicale», dans J. A. Coleman et C. M. Scollen-Jimack (dir.), Rabelais in Glasgow, Glasgow, Glasgow University, 1984, p. 87-11.

8. Voir Aphthonios, Progymnasmata, dans Corpus rhetoricum, éd. et trad. M. Patillon, Paris, Les Belles Lettres, 2008, p. 147 : « La description des personnages ira de ce qui est premier à ce qui est dernier, c'est-à-dire de la tête aux pieds, [...] celle des temps et des lieux se tirera des choses environnantes ». L'exemple de l'acropole d'Alexandrie employé par le rhéteur d'Antioche pour illustrer le mouvement de la périégèse est éloquent : le regard descripteur se porte d'abord sur le sentier qui mène au monument, avant d'observer les marches qui permettent d'y accéder et d'arriver à l'intérieur du temple. Voir également la description du Tychaion de Libanios: «The Exercice in Description ", dans Libanius's Progymnasmata: Model Exercises in Greek Prose Composition and Rhetoric, C. A. Gibson (éd.), Atlanta, Society of Biblical Literature, 2008, p. 487-491.

9. P. Hamon, Du descriptif, Paris, Hachette, 1993, p. 147.

10. P. Eichel-Lojkine, Excentricité et humanisme. Parodie, dérision et détournement des codes à la Renaissance, Genève, Droz, 2002, p. 37.

11. Sur la question de l'authenticité du Cinquiesme livre, voir M. Huchon, Rabelais grammairien. De l'histoire du texte aux problèmes d'authenticité, Genève, Droz, 1981.

12. Dans la définition qu'il propose de l'ekphrasis, Nicolaos de Myra signale plutôt que ce progymnasma «s'efforce de transformer les auditeurs en spectateurs » (Progymnasmata, dans Les progymnasmata de Nicolaos de Myra dans la tradition versicolore des exercices préparatoires de rhétorique, éd. et trad. H. Fruteau de Laclos, Lille, Atelier national de reproduction des thèses, 1999, p. 202).

13. L'enargeia est la capacité du discours à « [mettre] sous les yeux avec évidence ce qu'il donne à connaître" (Aphthonios, Progymnasmata, op.cit., p. 147). La même définition apparaît chez Pseudo-Hermogène et Ælius Théon.

14. P. Hamon, Du descriptif, op. cit., p. 65.

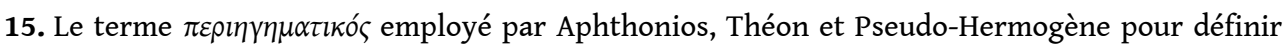
l'exposé descriptif est tiré du verbe $\pi \varepsilon p i \eta \gamma \varepsilon i ́ \sigma \theta \alpha l$ («tourner autour ») qui illustre le mouvement circumambulatoire propre à l'énoncé ekphrastique.

16. Vitruve, De l'architecture, P. Gros (éd.), Paris, Les Belles Lettres, 2015, p. 267.

17. L. Battista Alberti, L'Art d'édifier, éd. et trad. P. Caye et F. Choay, Paris, Seuil, 2004, p. 327.

18. Ibid., p. 325. 
19. Sur ce sujet, voir G. Mallary Masters, «Rabelais and Renaissance Figure Poems ", Genève, Droz (Études rabelaisiennes, vol. VIII), 1969, p. 53-68.

20. Ce passage est une réécriture de la description de la lampe dans le temple du Songe de Poliphile. Voir F. Colonna, Le Songe de Poliphile, G. Polizzi (éd.), J. Martin (trad.), Paris, Imprimerie nationale, 1994, p. 198-199 (nouvellement réédité chez Paris, Pocket, coll. « Agora », 2017).

21. Voir par exemple les trente ekphraseis qui composent le florilège attribué à Libanios, colligées et traduites par C.A.Gibson dans Libanios, "The Exercice in Description", Libanius's Progymnasmata: Model Exercises in Greek Prose Composition and Rhetoric, op.cit., p. 427-507. Sur Libanios, voir également B. Schouler, La tradition hellénique chez Libanios, Paris, Les Belles Lettres, 1984.

22. Pour une généalogie des éditions d'Aphthonios au $\mathrm{XVI}^{\mathrm{e}}$ siècle, voir J.-C. Margolin, « La rhétorique d'Aphthonius et son influence au XVI ${ }^{\mathrm{e}}$ siècle ", dans R. Chevalier (dir.), Colloque sur la rhétorique, Paris, Les Belles Lettres, 1979, p. 239-269.

23. Aphthonios, Progymnasmata, op. cit., p. 148-149.

24. Ibid., p. 151.

25. Certains récits rapportés par L Battista Alberti dans le De re cedificatoria font de Bacchus le premier bâtisseur des temples et des théâtres. Voir L. Battista Alberti, L'Art d'édifier, op. cit., p. 322-323 et p. 402.

26. Aphthonios, Progymnasmata, op. cit., p. 149.

27. T. Sébillet, « De l'Énigme ", Art poétique français [1548], dans Traités de poétique et de rhétorique de la Renaissance, F. Goyet (éd.), Paris, Le Livre de Poche, 1990, p. 134, cité dans R. Cappellen, «L'Énigme en prophétie, entre dualité auctoriale et pluralité interprétative (Gargantua, LVIII) ", Revue des littératures et des arts, $\mathrm{n}^{\circ} 17,2017$, note 47, en ligne : https://revues.univ-pau.fr/opcit/ 267.

28. N. Dauvois, «Le Bacchus du Cinquième Livre ou la logique de la description (chapitres XxxvIIXXXIX) », dans M. Simonin (dir.), Rabelais pour le XXI siècle, op. cit., p. 407. Voir aussi Y. Bellenger, " "Paindre à son plaisir". Les belles emblématures du temple de Bacbuc », dans F. Giacone (dir.), Le Cinquiesme Livre. Actes du colloque international de Rome (16-19 octobre 1998), Genève, Droz (Études rabelaisiennes, vol. XL), 2001, p. 291-301.

29. Nous empruntons ici la terminologie employée par Louis Richeome dans Tableaux sacrez des figures mystiques du très auguste sacrifice et sacrement de l'Eucharistie, Paris, L. Sonnius, 1609. Sur ce sujet, le lecteur consultera avec profit l'étude consacrée à cet ouvrage par R. Romagnino dans Théorie(s) de l'ecphrasis, op. cit., p. 131-140.

30. Philostrate, La Galerie de tableaux, introduction de P. Hadot, éd. F. Lissarrague, Paris, Les Belles Lettres, 2013. Les descriptions sonores sont également récurrentes dans les exercices de composition attribués à Libanios. Dans l'une de ces ekphraseis, le rhéteur d'Antioche décrit par exemple une scène de guerre où un soldat captif échange avec son ravisseur, où la cavalerie lance un cri martial et l'armée entonne un chant de victoire. Nous renvoyons par exemple au premier exercice du compendium de Libanios: «Description 1: an infantry battle» (Libanius's Progymnasmata : Model Exercises in Greek Prose Composition and Rhetoric, op. cit., p. 431).

31. R. Romagnino, Théorie(s) de l'ecphrasis, op. cit., p. 78.

32. Cette typologie est mentionnée dans les Progymnasmata d'Ælius Théon (éd. et trad. M. Patillon, Paris, Les Belles Lettres, 2002, p. 67) : "On a aussi des descriptions de "manières", comme celles qui décrivent les divers modes de production des mobiliers, des armes, des machines. Par exemple, la Fabrication des armes chez Homère et chez Thucydide le retranchement des Platéens ou la construction des machines. »

33. Cette ekphrasis est une réécriture de la description du mouvement des portes jumelles du temple du Songe de Poliphile qui s'ouvrent « sans aucun bruit sinon avec un doux et plaisant son ». Voir F. Colonna, Le Songe de Poliphile, op. cit., p. 202-203. Jean Martin a procédé à des adaptations majeures de l'Hypnerotomachia Poliphili dans sa traduction de 1546. Dans le texte-source, on 
remarque une instance et une valorisation extrême du sens de la vue, absentes de la traduction de Jean Martin et surtout du texte rabelaisien. Or, ce décalage entre le texte de Colonna et la description du Cinquiesme livre renforce notre propos: la mise en valeur de la vue est progressivement évacuée dans l'adaptation de cette ekphrasis par Rabelais qui procède à la « désagrégation » progressive du sens visuel au profit de l'ouïe.

34. F. Colonna, Le Songe de Poliphile, op.cit., p. 174-175, pour le chapitre xxxviII du CL; L. de Samosate, "Prolalia ou Dionysos ", dans éd. et trad. A.-M. Ozanam, Euvres complètes, Paris, Les Belles Lettres, 2018, p. 19-23, pour le chapitre XxxIx. Voir O. Pédeflous, Dans l'Atelier de Rabelais. Des recherches philologico-antiquaires à l'archéologie de la geste de Pantagruel, thèse de doctorat, Paris, Paris IV-Sorbonne, 2013 ; et R. Menini, Rabelais altérateur. "Graeciser en François », Paris, Classiques Garnier, 2014, p. 400-417.

35. Comme le soutient avec justesse Pierre Goumarre dans "Rabelais ou le regard humilié » (Littératures, $\mathrm{n}^{\circ} 6,1982$, p. 19) : «Pour Rabelais, l'organe sensoriel suprême, l'organe privilégié de la pensée, ce n'est pas l'œil, mais l'oreille » et «ce n'est pas l'œil, mais l'oreille, qui permet d'apprendre et de comprendre ». Même constat de la prédominance de l'ouie liée à l'expression de la parole dans A. Belleau, Notre Rabelais, Montréal, Boréal, 1990, p. 84. Dans un article de 2001, Olivier Millet fait bien voir que la progression des sonorités dans cette initiation "autre", préparée par les épisodes précédents, culmine dans la partie finale du Cinquiesme livre: voir O. Millet, «Le bruit et la musique dans le Cinquiesme livre », dans F. Giacone (dir.), Le Cinquiesme Livre, op. cit., p. 251-264.

\section{AUTEURS}

\section{DIANE DESROSIERS}

Université McGill

\section{OLIVIER SÉGUIN-BRAULT}

Université du Québec à Rimouski 